\title{
REORGANIZAÇÃo dO AUTOCONHECIMENTO E ADAPTAÇÃO PSICOLÓGICA NA ADOLESCÊNCIA
}

\author{
Maria João Carapeto \\ Universidade de Évora, Escola de Ciências Sociais \\ Departamento de Psicologia \\ mjoaocarapeto@gmail.com
}

Fecha de Recepción: 8 Mayo 2019

Fecha de Admisión: 25 Septiembre 2019

\section{RESUMO}

A reorganização do autoconhecimento tem sido considerada um dos temas mais importantes do desenvolvimento psicológico na adolescência. Considerando o autoconhecimento como teoria (ou teorias) pessoal com funções adaptativas, estas reorganizações podem ser vistas como movimentos adaptativos face às numerosas transformações primárias da adolescência. De um modo geral, dispersas na literatura, encontram-se referências a transformações físicas e a uma diversidade de novos contextos sociais com os quais 0 adolescente se depara e que requerem dele a construção de novas teorias, novos guiões pessoais para a sua atuação no mundo. É também referida na literatura a emergência de novas habilidades cognitivas e a possibilidade de construções cognitivas mais sofisticadas e flexíveis. Neste trabalho procede-se a uma revisão não sistemática de algumas das mais importantes transformações físicas, cognitivas e sociais da adolescência, e sua relação com mudanças no autoconhecimento. São abordados: (a) a emergência da sexualidade genital e as alterações da forma e do tamanho do corpo, e sua importância na construção de um autoconceito sexual e em alterações na imagem corporal; (b) as expetativas sociais relativamente aos adolescentes que contribuem para a criação de um sentido de identidade pessoal, bem como a circulação por um número crescente de contextos sociais favorece a construções de uma diversidade de eus relacionais (ou de eus em diferentes papeis sociais), e a influência que as redes sociais têm na construção de um "eu embrulho"; (c) a emergência de habilidades cognitivas abstratas, que possibilitam a diferenciação de múltiplos eus ou o crescimento das autodiscrepâncias (e.g., eu real - eu ideal), mas dão também lugar a conflitos no seio do self, antes de se poder alcançar um autoconhecimento mais coerente. É também sublinhada a interligação entre estas transformações (corporais, sociais e cognitivas) na construção do autoconhecimento.

Palavras chave: adolescência; autoconceito; puberdade; desenvolvimento cognitivo; contextos sociais; identidade. 


\title{
REORGANIZAÇÃO dO AUTOCONHECIMENTO E ADAPTAÇÃO PSICOLÓGICA NA ADOLESCÊNCIA
}

\begin{abstract}
Reorganization of self-knowledge and psychological adaptation in adolescence. The reorganization of self-knowledge has been considered one of the most important issues of psychological development in adolescence. Considering self-knowledge as personal theory (or theories) with adaptive functions, these reorganizations can be seen as adaptive movements before a diversity of primary transformations of adolescence. Dispersed in the literature, there are references to adolescents facing physical transformations and a diversity of new social contexts, which require from them the construction of new theories, new personal scrips for their action in the world. Also mentioned in the literature is the emergence of new cognitive skills and the possibility of more sophisticated and flexible cognitive construing. In this work we present a non-systematic review of some of the most important physical, cognitive and social transformations of adolescence and their relationship to changes in self-knowledge. The following issues are addressed: (a) the emergence of genital sexuality and changes in body shape and size, and their importance in building a sexual self-concept and in promoting changes in body image; (b) social expectations for adolescents that contribute to the creation of a sense of personal identity, the circulation through an increasing number of social contexts that favor the construction of a diversity of relational selves (or selves in different social roles), and the influence that social networks have on the construing of a "package self "; (c) the emergence of abstract cognitive abilities, which enable the differentiation of multiple selves or the growth of self-discrepancies (e.g., Actual - Ideal self), but also give rise to conflicts within the self, before a more coherent self-knowledge can be achieved. The interconnection between these transformations (physical, social and cognitive) in the construction of self-knowledge is also highlight.
\end{abstract}

Keywords: adolescence; self-concept; puberty; cognitive development; social contexts; identity.

\section{INTRODUÇÃO}

A reorganização do autoconhecimento tem sido reconhecida como tema central no desenvolvimento dos adolescentes, como ilustra a literatura sobre formação da identidade (e.g., Erikson, 1959; Meeus, 2011), autoconceito (e.g., Harter, 2012), autoestima (e.g., Chung, Hutteman, van Aken, \& Denissen, 2017), autodiscrepâncias (e.g., Higgins, 1989), internalização do eu ideal (e.g., Zentner \& Renaud, 2007), conflitos intrapessoais (e.g., Carapeto \& Feixas, 2019), entre outros.

Recentemente tem vindo a emergir algum consenso sobre as funções adaptativas do autoconhecimento. De modo geral, o autoconhecimento é entendido como teoria pessoal (ou teorias) que a pessoa vai construindo a partir da sua experiência, de modo a poder antecipar os acontecimentos do seu mundo e inspirar-Ihe atuações adequadas (adaptativas) em cada momento ou situação, bem como maximizar o seu bem-estar psicológico (e.g., Epstein, 1973; Erikson, 1959; Harter, 2012; Kelly, 1955). Neste contexto, as reorganizações do autoconhecimento que ocorrem na adolescência podem ser vistas, em boa parte, como movimentos adaptativos face à quantidade e diversidade de novidades e transformações (físicas, cognitivas, sociais, etc.) com que 0 adolescente se depara e que ele próprio gera. Apresenta-se neste trabalho uma breve revisão não sistemática da literatura sobre algumas das mais importantes alterações físicas, sociais e cognitivas da adolescência no mundo atual e suas relações com a reorganização do autoconhecimento neste período da vida.

\section{TRANSFORMAÇÕES FíSICAS DA ADOLESCÊNCIA E REORGANIZAÇÃO DO AUTOCONHECIMENTO}

A puberdade pode ser definida como uma série coordenada de mudanças neuro-hormonais e crescimento físico que formam o núcleo da transição da infância para a adolescência (Susman \& Dorn, 2009). Entre as transformações corporais encontram-se o surto de crescimento (alterações na altura e no peso), o desenvolvimento das características sexuais primárias (ovários, testículos) 
e secundárias (e.g., pelos púbicos, desenvolvimento dos seios e dos órgãos genitais masculinos) (Simmons, \& Blyth, 2017; Susman \& Dorn, 2009). Têm merecido também um interesse crescente as alterações ao nível da estrutura e do funcionamento do cérebro (Páus, 2009).

As alterações no tamanho e na forma do corpo bem como a emergência da sexualidade genital são acontecimentos dramáticos, especialmente no início da adolescência, e são recebidas com alegria e orgulho, mas também com medo e vergonha, por muitos adolescentes. Estes acontecimentos desencadeiam uma série de reorganizações do autoconhecimento, como por exemplo ao nível do esquema e da imagem corporal e da construção de sexualidade como nova dimensão no conhecimento de si mesmos (Canals, 2005; Coleman, 2011; Harter, 2012; Simmons \& Blytg, 2017; Schaffhuser, Allemand, \& Schwarz, 2017).

Vários autores referem que, inicialmente, as autodescrições que os adolescentes fazem de si próprios estão particularmente centradas em características físicas mas que, com o avançar na adolescência, as características de tipo psicológico ou social ganham espaço (Coleman, 2011; Oosterweggel \& Oppenheimer, 1993). No entanto, a satisfação com a aparência física revela-se sempre um contributo mais importante para a autoestima global do que a satisfação com as características autopercebidas noutros domínios da vida (escolar, social, etc.) (o que também acontece na infância e na idade adulta) (Harter, 2012).

Tem-se verificado também que a satisfação com a imagem corporal diminui ao longo da escolaridade, especialmente nas raparigas, que se percepcionam de modo significativamente mais desfavorável que os rapazes em dimensões como a aparência física ou as competências desportivas (Harter, 2012). Os rapazes parecem focar-se mais nos aspetos positivos do seu corpo e tendem a analisar a informação de modo mais favorável ao self (Ricciardelli \& McCabe, 2011; Wertheim \& Paxton, 2011).

Entre as caraterísticas especificas do corpo que mais frequentemente são motivo de insatisfação estão o peso e a forma do corpo, para as meninas (Wertheim \& Paxton, 2011), e a musculosidade e outras caraterísticas associadas às competências desportivas (força, velocidade, altura, etc.), para os rapazes (Ricciardelli \& McCabe, 2011). Uma boa parte das raparigas referem gostar de ser mais magras e essa proporção aumenta na adolescência; já os rapazes apresentam um padrão de manutenção, ou até de melhoria, da imagem corporal (Inchley et al., 2016).

Embora as alterações rápidas do corpo possam levar a desajustamentos ao nível da imagem corporal, estes parecem dever-se mais frequentemente à discrepância entre as caraterísticas corporais autopercebidas pelos adolescentes e 0 seu corpo ideal. E ganham aqui relevância os padrões de beleza veiculados na sociedade e a comparação social com pares (Wertheim \& Paxton, 2011). Outros estudos destacam que, mais do que as próprias transformações físicas, é o momento da maturação, tardia nos rapazes e precoce nas raparigas, que coloca os maiores desafios aos adolescentes (Coleman, 2011; Ricciardelli \& McCabe, 2011; Susman \& Dorn, 2009; Wertheim \& Paxton, 2011).

A emergência da sexualidade genital é outro impulsionador de reorganizações do autoconhecimento na adolescência. Um dos primeiros a propô-lo foi Sigmund Freud (1924), para quem a vida instintual dos adolescentes fica subordinada aos órgãos genitais e vários fenómenos pubertários (como a acrescida estimulação das zonas erógenas, 0 atingir da primazia genital e a intensificação da líbido) encaminham o adolescente para a busca de objetos sexuais adequados, que não os objetos edipianos incestuosos. 0 aumento dos conflitos instintuais vividos pelo adolescente ativa vários mecanismos de defesa para promover o reajustamento da personalidade, como a repressão, a formação reativa e a sublimação.

Numa perspectiva diferente, mais recentemente tem-se vindo a falar da construção de um auto- 
conceito sexual como tarefa desenvolvimental da adolescência. Trata-se de um construto multidimensional que se refere às percepções e sentimentos, positivos e negativos, de uma pessoa, acerca de si mesma como ser sexual (Snell, 1998). Alguns estudos com adolescentes têm vindo a destacar as dimensões de abertura para a sexualidade (reconhecimento do prazer sexual ou da excitação sexual, bem como um sentimento de direito ao exercício de atividades sexuais especificas), estima sexual (avaliações positivas da sexualidade pessoal, incluindo avaliações de pensamentos, sentimentos e comportamentos sexuais, bem como percepções do corpo no contexto sexual) e ansiedade sexual (tensão, desconforto e outras avaliações negativas relativamente a aspetos da sua própria vida sexual) (e.g., Hensel, Fortenberry, O'Sullivan, \& Orr, 2011). 0 autoconceito sexual evolui ao longo da adolescência de modo consistente no sentido de diminuição da reserva e da ansiedade e de maior conforto pessoal com a sexualidade e o comportamento sexual. Verificou-se que algumas das dimensões emergem no início da adolescência, antes mesmo de qualquer contacto sexual, que a prática sexual influencia a construção dos autoconceitos sexuais, e que estes regulam 0 comportamento sexual presente e futuro (Hensel et al., 2011).

\section{CONTEXTOS SOCIAIS DA ADOLESCÊNCIA E REORGANIZAÇÃO DO AUTOCONHECIMENTO}

As abordagens socioculturais do autoconhecimento (e.g., Baumeister \& Muraven, 1996; Gergen, 1991) defendem que as sociedades têm um papel muito importante na promoção, explícita ou implícita, de determinados modelos de eu, de ser pessoa ou de organização do autoconhecimento. Um modelo de eu emerge por ser aqueles que melhor prepara os indivíduos para participarem nessa sociedade e nela se realizarem adaptativamente.

Neste âmbito, uma das teorias mais influentes é ainda a teoria do desenvolvimento psicossocial de Erikson (1959), que vê a adolescência como uma fase em que a prontidão maturacional e as instituições sociais se combinam para promover a formação da identidade ao nível do indivíduo. A sociedade e seus representantes esperam que 0 adolescente defina projetos profissionais e prepare a sua autonomia face à família original e a construção de uma nova família. De acordo com a teoria, 0 adolescente vai procedendo à integração das múltiplas identificações da infância, explorando as oportunidades existentes no ambiente e formando a sua própria identidade. Construída a identidade, o indivíduo passa a dispor de uma formulação pessoal do seu papel social (ou papéis) e de um guião para se cumprir em sociedade para a vida toda. Em apoio desta hipótese, a investigação tem mostrado que a exploração de novas possibilidades de identidade aumenta ao longo da adolescência, que os compromissos identitários aumentam à medida que se entra na idade adulta e que nessa fase estes compromissos parecem associados a melhor bem-estar (Meeus, 2011).

Gergen (1991) defende que um modelo de eu constante e estável, ao longo do tempo e nas diferentes situações, como o descrito por Erikson, pode ter sido adaptativo nas sociedades industriais, que apresentavam lento débito de mudança, mas deixou de o ser nas sociedades pós-industriais em que vivemos. Estas sociedades, caraterizadas pela mudança rápida, pela globalização e pela incerteza, provavelmente carecem e estimulam a construção de um repertório de múltiplos eus relacionais, deixando a pessoa mais preparada para transitar flexivelmente nos diferentes contextos sociais da atualidade.

Este ambiente vertiginoso com que as sociedades atuais são descritas tem algum paralelo na experiência quotidiana dos adolescentes, que transitam entre uma multiplicidade de contextos sociais na sua vida. Terminado o primeiro ciclo do ensino básico em regime de monodocência, os adolescentes (em Portugal, desde $05^{0}$ ano) passam a ter uma escola organizada em torno de diversas disciplinas científicas, diversos professores, diversos espaços. Além disso, nas suas agendas semanais entram também uma variedade de novas tarefas como atividades desportivas, aulas privadas sobre as matérias escolares, ou outras atividades vistas como de enriquecimento extracurri- 
cular (música, línguas, etc.). Por outro lado, a família tradicional tem vindo a dar lugar a diversos formatos de família (ex., pertença a duas famílias, decorrente do divórcio dos pais), desafiando de novos modos a adaptação de crianças e adolescentes (Golombok, \& Tasker, 2015).

Além disso, os adolescentes passam mais tempo com os seus pares, sozinhos no seu quarto ou na internet (jogando ou participando em redes sociais), e menos tempo com os pais, sob a sua supervisão ou de outros adultos (Ferreira et al., 2017; Smetana, Robinson, \& Rote, 2014). Têm assim mais autonomia na gestão do seu comportamento e na tomada de decisões no seu quotidiano.

Alguns estudos têm procurado compreender 0 impacto de diferentes contextos de vida no desenvolvimento do autoconhecimento dos adolescentes, como por exemplo o contexto familiar (e.g., Syed \& Seiffge-Krenke, 2013), a transição escolar (e.g., Schaffhuser, Allemand \& Schwarz, 2017), ou o envolvimento nas redes sociais da internet (Valkenburg \& Peter, 2011). E alguma investigação da área cognitivo-desenvolvimentista interessou-se pelo desenvolvimento de múltiplos eus na adolescência (e.g., Fisher \& Kennedy, 1997; Harter, 2012), como se retomará adiante. No entanto, contrapõe-se também que, mais do que nunca, a possibilidade de construírem autoconhecimento bem integrado é importante para o bem-estar dos indivíduos (Harter, 2012; McAdams \& Maclean, 2013; Meeus, 2011).

Um dos fenómenos da atualidade cujo efeito na organização do autoconhecimento dos adolescentes tem merecido atenção crescente, é a utilização da internet e, em particular, das redes sociais. Globalmente, a investigação sobre os efeitos da comunicação online na construção da identidade dos adolescentes não é consistente, exceto quanto a um efeito positivo na autoestima (e.g., Valkenburg \& Peter, 2011). Os estudos qualitativos de Gardner e Davies (2014) apontam efeitos positivos e negativos. Referem como benéficas as novas oportunidades de experimentação da identidade e de validação social do autoconhecimento. Mas assinalam a formação de identidades mais individualistas e narcisistas, centradas no desejável aos olhos dos outros, sugerindo identidades perfeitas, completadas, sem que qualquer exploração introspetiva tenha tido lugar (0 "eu embrulho", package self). Falam ainda de uma mentalidade app, para dizer que os jovens estão a construir modos de pensar algorítmicos que exigem soluções imediatas para os seus problemas e desejos, afugentando a experiência de ambiguidades ou incertezas.

\section{AVANÇOS COGNITIVOS DA ADOLESCÊNCIA E REORGANIZAÇÃO DO AUTOCONHECIMENTO}

Baseada na teoria de Piaget (Inhelder \& Piaget, 1958), a teoria das habilidades dinâmicas de Fischer (e.g., Fischer \& Bidell, 2006) tem-se revelado útil para compreensão do desenvolvimento cognitivo ao longo da vida e do desenvolvimento do autoconhecimento na adolescência. Propõe que as habilidades cognitivas abstratas emergem na adolescência e que se desenvolvem ao longo de três níveis de complexidade crescente (abstrações individuais, mapeamento de abstrações e sistema de abstrações), cada um deles emergindo do anterior em momentos distintos da adolescência. Considera ainda que estes níveis cognitivos começam por surgir só em condições ótimas, i.e., quando há 0 apoio de outra pessoa cognitivamente mais competente.

Em parte baseada na teoria de Fischer, Harter (2012) descreve o desenvolvimento do autoconhecimento (conteúdos e organização) em três fases ao longo da adolescência, como resultado da necessidade de novos guiões para funcionar nos novos contextos sociais da adolescência e da disponibilidade de habilidades cognitivas mais sofisticadas.

$\mathrm{Na}$ fase inicial da adolescência os jovens são capazes de combinar as autorrepersentações da infância e assim formar abstrações (abstrações isoladas). Nesta fase, os adolescentes diferenciam cada vez mais as suas caraterísticas em função dos contextos relacionais em que participam (euem-relação-com-a-minha-mãe, eu-em-relação-com-a-minha-melhor-amiga, etc.) ou de diferentes 
domínios da vida (competência escolar, competência social, aparência física, comportamento, amizades íntimas, atração romântica, competências de trabalho), bem como a autoestima se diferencia em função dos contextos relacionais. Na fase intermédia da adolescência, os jovens passam a ser capazes de perceber contradições entre as características pessoais diferenciadas, ocasionando conflitos internos e a preocupação sobre a existência de eus falsos e sobre qual será o eu verdadeiro (Fischer \& Keneddy, 1997; Harter, 2012). No final da adolescência, as habilidades cognitivas de integração de abstrações estarão disponíveis e será possível construir um autoconhecimento mais coerente. Todavia, para o conseguir, os adolescentes mais velhos precisam frequentemente do apoio de uma pessoa mais madura (Fischer \& Kennedy, 1997; Harter, 2012).

Outra literatura mostra também a importância das novas habilidades cognitivas para diferenciar e integrar 0 autoconhecimento. Por exemplo, a investigação mostra uma distância crescente entre o Eu Real e o Eu Ideal (e.g., Glick \& Zigler, 1985). Esta diferenciação crescente tem sido interpretada também como parte da internalização de autoguias (especialmente o Eu Ideal) que ocorre na adolescência (e.g., Higgins, 1989; Zentner \& Renaud, 2007). A autodiscrepância Real-Ideal tem sido também considerada uma fonte de autoestima (menor discrepância, maior autoestima) (Ferguson, Hafen \& Laursen, 2010; Higgins, 1989; Pinquart, Silbereisen \& Wiesner, 2004).

Também a investigação sobre individuação e formação da identidade vai no mesmo sentido da diferenciação e integração crescentes (Blos, 1968; Erikson, 1959). Alguns estudos têm efetivamente mostrado que 0 sentido de ser diferente dos outros (van Doeselaar, Becht, Klimstra, \& Meeus, 2018), a exploração de diferentes identidades e a emergência de diferentes domínios identitários (Meeus, 2011) aumentam ao longo da adolescência. Contudo, no final da adolescência, a intervenção de habilidades integrativas que combinem essas diversas possibilidades psicossociais em compromissos identitários fortes parece ser importante para o bem-estar e a adaptação psicológica (Erikson, 1959; Meeus, 2011).

\section{CONCLUSÕES}

Foi abordado, de modo não exaustivo, como um conjunto de transformações primárias normativas (físicas, sociais e cognitivas) com que os adolescentes se deparam podem impulsionar reorganizações do autoconhecimento, considerando que este tem funções adaptativas.

Não se fique, no entanto com a ideia de que há uma relação biunívoca entre cada uma das possíveis transformações primárias e uma particular mudança no autoconhecimento. Como se foi evidenciando, as reorganizações do autoconhecimento podem ser mais bem entendidas como um esforço combinado perante alterações e novidades de diferentes tipos na vida dos adolescentes. Por exemplo, a construção de múltiplos eus-em-relação-com- poderá ser estimulada pela crescente diversidade de contextos relacionais no início da adolescência, mas depende também da disponibilidade das primeiras habilidades cognitivas abstratas (Harter, 2012).

Por outro lado, os avanços na reorganização do autoconhecimento podem trazer ganhos em flexibilidade e autonomia na atuação dos jovens nos seus contextos de vida, mas podem comportar também alguns riscos para a adaptação psicológica, mesmo que transitórios. Por exemplo, quando os avanços cognitivos permitem que os adolescentes percebam contradições no seio do seu autoconhecimento sem que as consigam ainda integrar, ou quando estabelecem ideais ambiciosos e difíceis de atingir num período razoável de tempo, é possível que surja sofrimento internalizado ou que a autoestima sofra (Carapeto \& Feixas, 2019). Alguns destes avanços na organização do autoconhecimento podem, pois, estar associados, pelo menos em parte, à agitação e tensão (storm and stress) que, segundo alguns autores (Arnett, 1999), caracteriza a adolescência.

Concluindo, a abordagem do autoconhecimento como adaptação às diversas alterações expe- 
rienciadas pelos adolescentes oferece, da nossa perspetiva, um quadro promissor para a investigação da adaptação na adolescência, bem como para a prática psicológica e educativa com adolescentes.

\section{REFERÊNCIAS BIBLIOGRÁFICAS}

Arnett, J. J. (1999). Adolescent Storm and Stress, Reconsidered. American Psychologist, 54, 317326.

Baumeister, R. F., \& Muraven, M. (1996). Identity as adaptation to social, cultural, and historical context. Journal of Adolescence, 19, 405-416.

Blos, P. (1968). Character Formation in Adolescence. The Psychoanalytic Study of the Child, 23, 245-263.

Canals, J. (2005). El impacto de la pubertad sobre el desarrollo del adolescente. In E. DomenèchLlaberia (Ed.), Actualizaciones en psicología y psicopatología de la adolescencia (págs. 39-52). Barcelona: Universitat Autonoma de Barcelona.

Carapeto, M. J. \& Feixas, G. (2019). Self-knowledge and depressive symptoms in late adolescence: A study using the repertory grid technique. Journal of Constructivist Psychology, 32:1, 81-97. https://doi.org/10.1080/10720537.2018.1433087

Chen, S., Boucher, H., \& Kraus, M. W. (2011). The relational self. In S. J. Schwartz, K. Luyckx \& V. L. Vignoles (Eds.), Handbook of identity theory and research (pp. 149-175). New York, N.Y.: Springer.

Chung, J. M., Hutteman, R., van Aken, M. A., \& Denissen, J. J. (2017). High, low, and in between: Self-esteem development from middle childhood to young adulthood. Journal of Research in Personality, 70, 122-133. https://doi.org/10.1016/j.jrp.2017.07.001

Coleman, J. C. (2011). The nature of adolescence (4th Ed). New York, NY: Psychology Press.

Crockett, L. J., \& Silbereisen, R. K. (2000) (Eds.). Negotiating adolescence in times of social change. Cambridge, U.K.: Cambridge University Press.

Domenèch-Llaberia, E. (Ed.) (2005). Actualizaciones en psicologia y psicopatologia de la adolescencia. Barcelona: Universitat Autonoma de Barcelona

Epstein, S. (1973). The self-concept revisited: Or a theory of a theory. American psychologist, 28(5), 404.

Erikson, E. (1959). Identity and the Life Cycle. New York: Norton.

Ferguson, G. M., Hafen, C. A., \& Laursen, B. (2010). Adolescent psychological and academic adjustment as a function of discrepancies between actual and ideal self-perceptions. Journal of Youth and Adolescence, 39(12), 1485-1497.

Ferreira, C., Ferreira, H., Vieira, M. J., Costeira, M., Branco, L., Dias, A., \& Macedo, L. (2017). Epidemiologia do uso de internet numa população adolescente e sua relação com hábitos de sono. Acta Médica Portuguesa, 30(7-8), 524-533.

Fischer, K. W., \& Bidell, T. R. (2006). Dynamic Development of Action and Thought. In W. Damon e R. M. Lerner (Eds.), Theoretical Models of Human Development. Handbook of Child Psychology (6 $6^{\text {th }}$ Ed, Vol 1, pp. 313-399). New York: Wiley.

Fischer, K. W., \& Kennedy, B. P. (1997). Tools for analysing the many shapes of development: the case of self-in-relationships in Korea. In E. Amsel \& K. A. Renninger (Eds.), Change and Development: Issues of Theory, Method and Application, The Jean Piaget Symposium Series (pp. 117-152). Mahwah, NJ: Lawrence Erlbaum.

Freud, S. (1924). Três Ensaios sobre a Teoria da Sexualidade. Lisboa: Livros do Brasil.

Gardner, H., \& Davis, K. (2013). The app generation: How today's youth navigate identity, intimacy, and imagination in a digital world. New Haven, CT: Yale University Press. 
Gergen, K. J. (1991). The Saturated Self: Dilemmas of Identity in Contemporary Life. New York: Basic Books.

Glick, M., \& Zigler, E. (1985). Self-image: a cognitive-developmental approach. In R. L. Lehary (Ed.), The development of the self (pp. 1-53). London: Academic Press.

Golombok, S. \& Tasker, F. (2015). Socioemotional Development in Changing Families. In R. M. Lerner (Ed.), Handbook of child psychology and developmental science (7th Ed.) (Vol. 3, pp. 419-465). Wiley.

Harter, S. (2012). The construction of the self: Developmental and sociocultural foundations $\left(2^{\text {nd }}\right.$ Ed.). New York, NY: Guilford Publications.

Hensel, D. J., Fortenberry, J. D., O'Sullivan, L. F., \& Orr, D. P. (2011). The developmental association of sexual self-concept with sexual behavior among adolescent women. Journal of adolescence, 34(4), 675-684.

Higgins, E. T. (1989). Continuities and discontinuities in self-regulatory and self-evaluative processes: a developmental theory relating self and affect. Journal of Personality, 57, 407-444.

Hurrelmann, K. (1994). Introduction: Interdisciplinary and International Approaches to Research on Adolescence. In K. Hurrelmann (Ed), International Handbook of Adolescence (pp. 1-332). Westport, Connecticut \& London: Greenwood Press.

Inchley, J. et al. (Eds.) (2016). Growing up unequal: gender and socioeconomic differences in young people's health and well-being. Health behaviour in school-aged children (HBSC) study: international report from the 2013/2014 survey. Copenhagen, Denmark: World Health Organization Regional Office for Europe.

Inhelder, B., \& Piaget, J. (1958). The Growth of Logical Thinking From Childhood to Adolescence. New York: Basic Books.

Kelly, G. A. (1955). The Psychology of Personal Constructs. New York: Norton \& Company.

McAdams, D. P., \& McLean, K. C. (2013). Narrative identity. Current directions in psychological science, 22(3), 233-238.

Meeus, W. (2011). The study of adolescent identity formation 2000-2010: A review of longitudinal research. Journal of Research on Adolescence, 21(1), 75-94. https://doi.org/10.1111/j.15327795.2010.00716.x

Oosterwegel, A., \& Oppenheimer, L. (1993). The Self-System. Developmental Changes Between and Within Self-Concepts. Hillsdale, NJ: Erlbaum.

Páus, T. (2009). Brain development. In R. M. Lerner and L. Steinberg (Eds.), Handbook of Adolescent Psychology, Individual Bases of Adolescent Development (Vol. 1) (pp. 95-115). John Wiley \& Sons.

Pinquart, M., Silbereisen, R. K., \& Wiesner, M. (2004). Changes in discrepancies between desired and present states of developmental tasks in adolescence: a 4-process model. Journal of Youth and Adolescence, 33, 467-477.

Ricciardelli, L. A., \& McCabe, M. P. (2011). Body Image Development in Adolescent boys. In Thomas F. Cash and Linda Smolak (Eds.), Body image. A handbook of science, practice, and prevention (pp. 85-92). New York, NY: Routledge.

Schaffhuser, K., Allemand, M., \& Schwarz, B. (2017). The Development of Self-Representations During the Transition to Early Adolescence: The Role of Gender, Puberty, and School Transition. The Journal of Early Adolescence, 37(6), 774-804. https://doi.org/10.1177/0272431615624841

Simmons, R. G., \& Blyth, D. A. (2017). Moving into Adolescence: The impact of Pubertal Change and School Context. New York, N.Y.: Routledge.

Smetana, J. G., Robinson, J., \& Rote, W. M. (2014). Socialization in adolescence. In J. E. Grusec and 
P. D. Hastings (Eds.), Handbook of Socialization: Theory and Research (2nd Ed.) (pp. 60-84). Guilford.

Snell, W. E. (1998). The multidimensional sexual self-concept questionnaire. In C. M. Davis, W. L. Yarber, R. Bauserman, G. Schreer and S.L.Davis (Eds.), Handbook of sexuality-related measures (pp. 521-524). Sage.

Susman, E.J. \& Dorn, L.D. (2009). Puberty: its role in development. In R. M. Lerner and L. Steinberg (Eds.), Handbook of Adolescent Psychology, Individual Bases of Adolescent Development (Vol. 1) (pp. 116-151). John Wiley \& Sons.

Syed, M., \& Seiffge-Krenke, I. (2013). Personality development from adolescence to emerging adulthood: Linking trajectories of ego development to the family context and identity formation. Journal of personality and social psychology, 104(2), 371.

Valkenburg, P. M., \& Peter, J. (2011). Online communication among adolescents: An integrated model of its attraction, opportunities, and risks. Journal of adolescent health, 48(2), 121-127.

Van Doeselaar, L., Becht, A. I., Klimstra, T. A., \& Meeus, W. H. (2018). A review and integration of three key components of identity development. European Psychologist, 23, 278-288. https://doi.org/10.1027/1016-9040/a000334.

Wertheim, E. H., \& Paxton, S. J. (2011). Body Image Development in Adolescent Girls. In Thomas F. Cash and Linda Smolak (Eds.), Body image. A handbook of science, practice, and prevention (pp. 76-84). New York, NY: Routledge.

Zentner, M., \& Renaud, 0. (2007). Origins of adolescents' ideal self: an intergenerational perspective. Journal of Personality and Social Psychology, 92, 557-574. http://dx.doi.org/10.1037/00223514.92.3.557 
